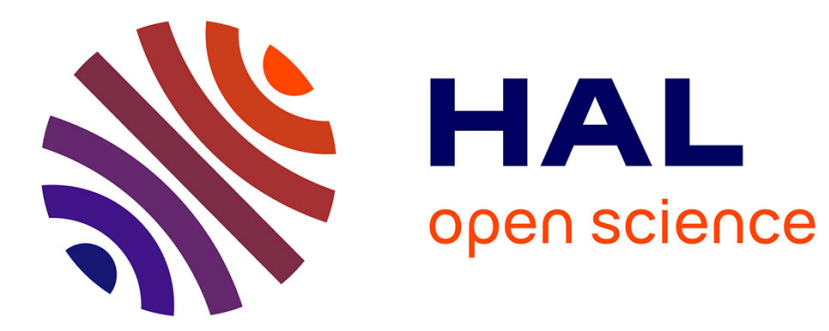

\title{
Near-forward Raman study of a phonon-polariton reinforcement regime in the $\mathrm{Zn}(\mathrm{Se}, \mathrm{S})$ alloy
}

R. Hajj Hussein, O. Pagès, F. Firszt, A. Marasek, W. Paszkowicz, A. Maillard, L. Broch

\section{- To cite this version:}

R. Hajj Hussein, O. Pagès, F. Firszt, A. Marasek, W. Paszkowicz, et al.. Near-forward Raman study of a phonon-polariton reinforcement regime in the $\mathrm{Zn}(\mathrm{Se}, \mathrm{S})$ alloy. Journal of Applied Physics, 2014, 116 (8), 10.1063/1.4893322 . hal-01516065

\section{HAL Id: hal-01516065 \\ https://hal.univ-lorraine.fr/hal-01516065}

Submitted on 11 May 2017

HAL is a multi-disciplinary open access archive for the deposit and dissemination of scientific research documents, whether they are published or not. The documents may come from teaching and research institutions in France or abroad, or from public or private research centers.
L'archive ouverte pluridisciplinaire HAL, est destinée au dépôt et à la diffusion de documents scientifiques de niveau recherche, publiés ou non, émanant des établissements d'enseignement et de recherche français ou étrangers, des laboratoires publics ou privés. 


\section{Near-forward Raman study of a phonon-polariton reinforcement regime in the $\mathrm{Zn}(\mathrm{Se}, \mathrm{S})$}

alloy

R. Hajj Hussein, O. Pagès', F. Firszt, A. Marasek, W. Paszkowicz, A. Maillard, and L. Broch

Citation: Journal of Applied Physics 116, 083511 (2014); doi: 10.1063/1.4893322

View online: http://dx.doi.org/10.1063/1.4893322

View Table of Contents: http://aip.scitation.org/toc/jap/116/8

Published by the American Institute of Physics

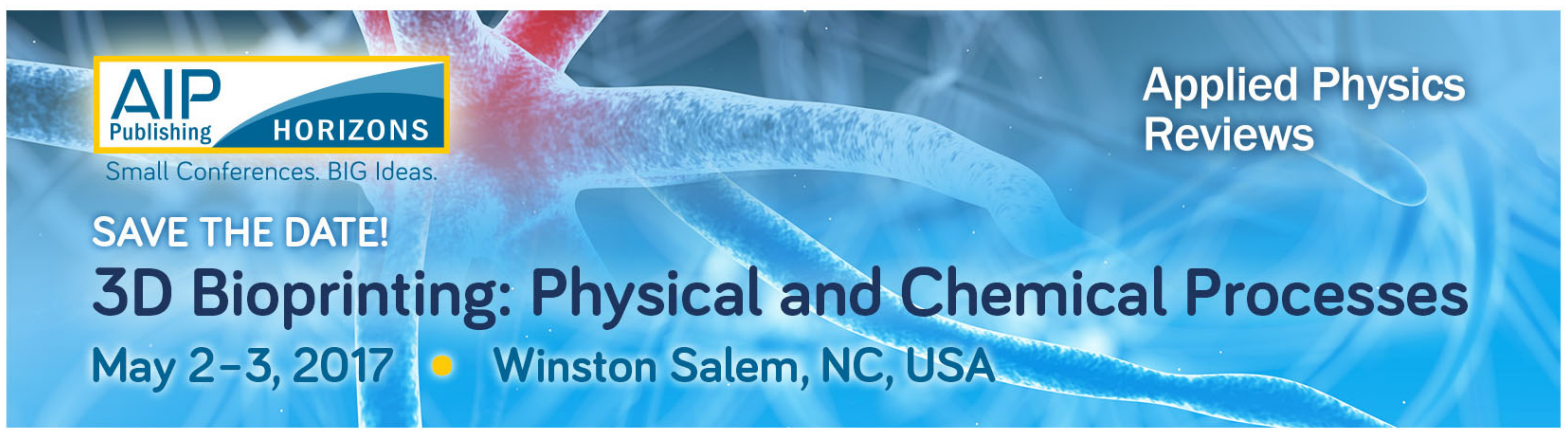




\title{
Near-forward Raman study of a phonon-polariton reinforcement regime in the $\mathrm{Zn}(\mathrm{Se}, \mathrm{S})$ alloy
}

\author{
R. Hajj Hussein, ${ }^{1}$ O. Pagès, ${ }^{1, a)}$ F. Firszt, ${ }^{2}$ A. Marasek, ${ }^{2}$ W. Paszkowicz, ${ }^{3}$ A. Maillard, ${ }^{4}$ \\ and L. Broch ${ }^{1}$ \\ ${ }^{1}$ LCP-A2MC, Institut Jean Barriol, Université de Lorraine, 1 Bd. Arago, 57078 Metz, France \\ ${ }^{2}$ Institute of Physics, N. Copernicus University, 87-100 Toruń, Poland \\ ${ }^{3}$ Institute of Physics, Polish Academy of Sciences, 02-668 Warsaw, Poland \\ ${ }^{4}$ LMOPS, Université de Lorraine - Sup'elec, 2, rue Edouard Belin, 57070 Metz, France
}

(Received 25 June 2014; accepted 5 August 2014; published online 27 August 2014)

\begin{abstract}
We investigate by near-forward Raman scattering a presumed reinforcement of the (A-C,B-C)mixed phonon-polariton of a $\mathrm{A}_{1-\mathrm{x}} \mathrm{B}_{\mathrm{x}} \mathrm{C}$ zincblende alloy when entering its longitudinal optical-like (LO-like) regime near the Brillouin zone centre $\Gamma$, as predicted within the formalism of the linear dielectric response. A choice system to address such issue is $\mathrm{ZnSe}_{0.68} \mathrm{~S}_{0.32}$ due to the moderate dispersion of its refractive index in the visible range, a sine qua non condition to bring the phononpolariton insight near $\Gamma$. The $L O$-like reinforcement regime is actually accessed by using the $633.0 \mathrm{~nm}$ laser excitation, testified by the strong emergence of the (Zn-Se,Zn-S)-mixed phononpolariton at ultimately small scattering angles. (C) 2014 AIP Publishing LLC.

[http://dx.doi.org/10.1063/1.4893322]
\end{abstract}

\section{INTRODUCTION}

Due to the polarity of the chemical bonding in such a ionic crystal as a zincblende $\mathrm{AB}$ semiconductor compound, the long-wavelength ( $\Gamma$-like, $q \sim 0$ ) transverse optical $(T O)$ phonon, corresponding to anti-phase displacement of the intercalated A-like and B-like $f c c$ sublattices (mechanical character), is likely to be accompanied by a macroscopic electric field. ${ }^{1}$ The latter is transversal to the direction of propagation, thus identical in nature to that carried by a pure electromagnetic wave, namely a photon. Now, due to the quasi vertical " $\omega$ vs. $q$ " dispersion of a photon at the scale of the Brillouin zone, the electromagnetic character of a TO mode can only emerge very close to $\Gamma$. The concerned $q$ values are of the order of one per ten thousands of the Brillouin zone size. ${ }^{2}$ At this limit, the electromagnetic and mechanical characters combine, conferring on a TO mode the status of a so-called phonon-polariton $(P P)$. For certain $q$ values, the $P P$ might acquire a dominant electromagnetic character, thus propagating at lightlike speeds. This stimulates interest in view of ultrafast (photon-like) signal processing at $\mathrm{THz}$ (phonon-like) frequencies. ${ }^{3}$

The " $\omega$ vs. $q$ " dispersion of $P P$ 's propagating in the bulk of various $\mathrm{AB}$ zincblende compounds have been abundantly studied, both experimentally and theoretically., ${ }^{2,4}$ In a nutshell, it can be grasped within four asymptotic behaviors, i.e., two photon-like ones ( $\omega$-related) and two phonon-like ones ( $q$-related). For large $q$ values, i.e., falling within few percent of the Brillouin zone size, as routinely accessible in a conventional backscattering Raman experiment (schematically operating in a "reflection mode," see below), a transverse electric field cannot propagate at $\mathrm{THz}$ (phonon-like) frequencies.

\footnotetext{
a) Author to whom correspondence should be addressed. Electronic mail: olivier.pages@univ-lorraine.fr.
}

This is because the considered $(\omega, q)$-domain falls far away from the natural " $\omega$ vs. $q$ " dispersion of a photon (quasi vertical). In such so-called $q_{\infty}$-regime, a TO mode thus reduces to a purely mechanical oscillator (abbreviated $P M-T O$ hereafter, deprived of electric field), whose frequency, noted $\omega_{T O}$, constitutes the first phonon-like asymptote, i.e., away from $\Gamma$. The frequency of the non-dispersive longitudinal optical $(L O)$ mode, noted $\omega_{L O}$, larger than $\omega_{T O},{ }^{10}$ defines the second phonon-like asymptote, near $\Gamma$ then, taking into account that the $T O$ and $L O$ modes are degenerate strictly at $\Gamma{ }^{11}$ Two remaining photon-like asymptotes determine limit $P P$-behaviors away from the (PM-TO)-LO resonance, as dictated by the static $\varepsilon_{0}\left(\omega \ll \omega_{T O}\right)$ and high-frequency $\varepsilon_{\infty}\left(\omega \gg \omega_{T O}\right)$ relative dielectric constants of the crystal. The strong $P P$ coupling occurs when the quasi vertical photon-like asymptotes cross the horizontal $P M-T O$ phonon-like ones. This gives rise to an anticrossing, resulting in two distinct $P P$ branches. The upper branch is phonon-like $(L O)$ when $q \rightarrow 0$ and photonlike $\left(\omega=q \times c \times \varepsilon_{\infty}^{-1}\right.$, where c represents the speed of light in vacuum) at $\omega \gg \omega_{T O}$, while the lower branch is photon-like $\left(\omega=q \times c \times \varepsilon_{\infty}^{-1}\right)$ at $\omega \ll \omega_{T O}$ and phonon-like in the $q_{\infty}$-regime (PM-TO). Note that the (PM-TO)-LO band is forbidden for the propagation of bulk $P P$ 's, only surface $P P$ 's can propagate therein.

An interesting question is how such $P P$ picture modifies for a multi-oscillator system such as a $A_{1-x} C_{x}$ zincblende alloy (A standing indifferently for a cation or an anion)? Bao and Liang provided a pioneering theoretical insight into the " $\omega$ vs. $q$ " $P P$-dispersion of various $\mathrm{AB}_{1-\mathrm{x}} \mathrm{C}_{\mathrm{x}}$ zincblende alloys. ${ }^{12,13}$ As a starting point they assumed a basic twomode $[1 \times(\mathrm{A}-\mathrm{C}), 1 \times(\mathrm{B}-\mathrm{C})] P M-T O$ pattern behind the $P P^{\prime} \mathrm{s}$, as explained within the well-known modified-randomelement-isodisplacement (MREI) model. ${ }^{14}$ Besides the lower (noted $L P$ ) and upper (noted $U P$ ) $P P$ branches, assimilating to those of a pure compound as described above, ${ }^{2,4-9}$ an 
intermediary (A-C, B-C)-mixed $P P$ branch (noted $M P$, in abbreviation of medium phonon-polariton) was predicted by Bao and Liang. The latter $M P$ branch is distinct in nature from the former $(L P, U P)$ parent-like ones in that it exhibits an overall S-like shape governed by two phonon-like (horizontal) asymptotes only, i.e., the higher $P M-T O$ frequency in the $q_{\infty}$-regime, say the BC-like one, and the lower $L O$ frequency near $\Gamma$, the AC-like one then, with a photon-like regime in between approximately corresponding to the inflexion of the S-like branch. ${ }^{12,13}$ As such, its dispersion covers the gap between the natural $A-C$ and $B-C$ vibration frequencies, possibly a considerable one depending on the alloy.

An example of such " $\omega$ vs. $q$ " MREI-like (two PM$T O$ 's) $P P$-dispersion, concerning the particular $\mathrm{ZnSe}_{0.68} \mathrm{~S}_{0.32}$ zincblende alloy, of interest in this work (see below), is given in Fig. 1, to fix ideas. The whole picture is governed by the PM-TO frequencies of the two $\mathrm{Zn}-\mathrm{Se}$ and $\mathrm{Zn}-\mathrm{S}$ oscillators, taken at $\sim 210$ and $\sim 285 \mathrm{~cm}^{-1}$, respectively (see below), from recent estimates by Vinogradov et al. (Ref. 15, see Fig. 3 therein). Besides, a basic rule is applied that the amount of oscillator strength available per bond at a given alloy composition scales as the corresponding bond fraction, i.e., as 0.68 for $\mathrm{Zn}-\mathrm{Se}$ and as 0.32 for $\mathrm{Zn}-\mathrm{S}$ for the considered alloy. The $\mathrm{Zn}-\mathrm{Se}$ and $\mathrm{Zn}-\mathrm{S}$ LO frequencies are accordingly derived. Further detail concerning the method used to calculate the $P P$-dispersion and the input parameters needed to implement such calculation is given below, at the occasion of a more accurate (three $P M-T O$ 's) description of the $\mathrm{ZnSe}_{0.68} \mathrm{~S}_{0.32} P P$-dispersion.

We have refined the description of the " $\omega$ vs. $q$ " $P P$ dispersion of an alloy beyond the MREI scheme at the occasion of a recent near-forward Raman study (schematically operating in a "transmission mode," see below) of the $\mathrm{Zn}_{0.67} \mathrm{Be}_{0.33} \mathrm{Se}$ zincblende alloy, characterized by a threemode $[1 \times(\mathrm{Zn}$-Se $), 2 \times(\mathrm{Be}-\mathrm{Se})] P M-T O$ pattern in the $q_{\infty}$-regime. Such three-mode pattern falls out of the scope of the MREI scheme, by construction. It was explained by

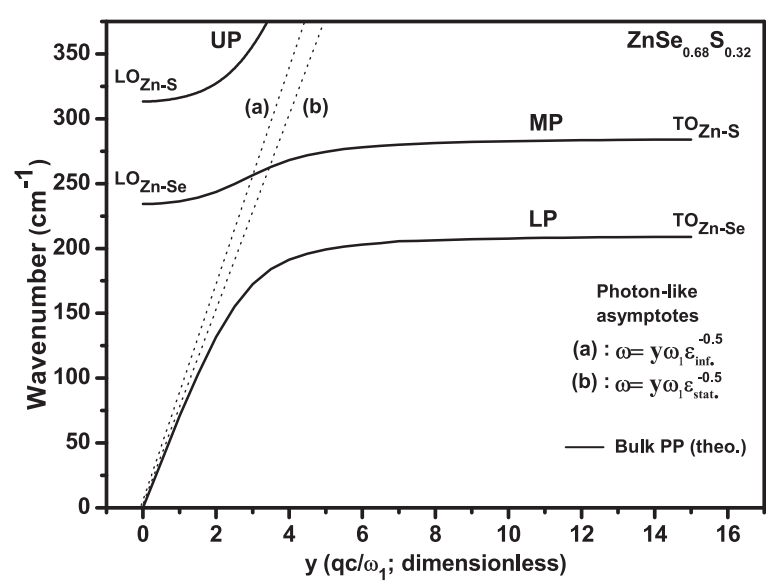

FIG. 1. $\mathrm{ZnSe}_{0.68} \mathrm{~S}_{0.32}$ ' $\omega$ vs. $q$ ' $P P$ dispersion calculated from Eq. (3) on the basis of a two-mode $[1 \times(\mathrm{Zn}-\mathrm{Se}), 1 \times(\mathrm{Zn}-\mathrm{S})]$ MREI-like description of the $P M$-TO's (abbreviated TO) in the $q_{\infty}$-regime, giving rise to a three-mode (lower- $L P$, medium- $M P$, upper- $U P$ ) phonon-polariton pattern. The $L O$ asymptotes near $\Gamma$ ( $L O$ labeling) and the photon ones (thin lines) far beyond (a) and far below (b) the (PM-TO)-LO phonon resonances are specified, for reference purpose. A more refined three-mode $[1 \times(\mathrm{Zn}-\mathrm{Se}), 2 \times(\mathrm{Zn}-\mathrm{S})]$ description is given in Fig. 7. introducing the phenomenological percolation model. ${ }^{16}$ In brief, this model distinguishes between the vibrations of the short (Be-Se) bonds depending on whether their local environment is more rich of one or the other ( $\mathrm{Zn}-\mathrm{Se}$ or Be-Se) species (1-bond $\rightarrow 2$-mode behavior). ${ }^{17}$ In this study, two intermediary (Zn-Se,Be-Se)-mixed MP's were revealed, and not only one as predicted within the MREI scheme. Each $M P$ relates to a particular BeSe-like $P M$-TO in the $q_{\infty}$-regime, and collapses with an S-like shape onto the $L O$ immediately underneath near $\Gamma$, consistently with the basic MREI trend (refer to Fig. 1). In particular, the lower $M P$, noted $M P^{-}$, attracts attention, for two reasons. First, in contrast with the upper $M P$, noted $M P^{+}$, which remains confined within the upper doublet of $P M-T O^{\prime}$ 's (BeSe-like in this case), $M P^{-}$may exhibit a considerable dispersion (covering the gap between the $\mathrm{Zn}$-Se and Be-Se vibration frequencies in this case), as discussed above within the MREI scheme. Second, as soon as entering the $P P$ regime, $M P^{-}$becomes dominant over $M P^{+}{ }^{16}$

The $q$-dependence of the $M P^{-}$Raman intensity constitutes per se an interesting issue. Though this mode has a basic $T O$ character throughout its whole dispersion, we anticipate variations in its Raman activity depending on whether $M P^{-}$ is more phonon-like, in reference to both asymptotic regimes, presumably corresponding to a large Raman activity, or more photon-like, in reference to the intermediary regime, in which the Raman activity is expected to be small-negligible. Based on our recent near-forward Raman study of $\mathrm{Zn}_{0.67} \mathrm{Be}_{0.33} \mathrm{Se}$ limited to the early stage of the $P P$ regime (a moderate $M P^{-}$ red-shift of $\sim 15 \mathrm{~cm}^{-1}$ was detected, representing less than ten percent of the total $M P^{-}$dispersion), we already know that the initial $q$-induced softening of $M P^{-}$goes with a progressive collapse of this mode, the sign that $M P^{-}$progressively enters the intermediary photon-like regime. ${ }^{16}$ With further reduction in $q$ the photon-like character should be lost at a certain stage, and near $\Gamma$, where $M P^{-}$eventually assimilates to a $L O,{ }^{12,13,16}$ the Raman scattering should reactivate, in principle. Summarizing, when progressing towards $\Gamma$, we expect that the initial $\left(P M-T O \rightarrow\right.$ photon) collapse of $M P^{-}$ should be relayed by a (photon $\rightarrow L O$ ) reinforcement at a certain stage. This points to a specific $P P$ feature of an alloy, yet unexplored, neither experimentally nor theoretically.

Interestingly, such a dependence of Raman efficiency in polaritons from matter-like to photon-like and again to matter-like has recently been observed for the $L O$ Raman scattering mediated via exciton-polaritons in a pure compound. ${ }^{18}$ However, in the cited work the Raman efficiency increases in the strong coupling regime (more photon-like) and decreases when the polariton takes a dominant excitonic (matter-like) character. Basically, we expect the opposite "matter vs. photon" interplay for the Raman efficiency in our case of a $T O$-like Raman scattering by the lower-medium phonon-polariton branch of an alloy.

In this work we tackle such issue both theoreticallywithin the formalism of the linear dielectric response, and experimentally — by applying the near-forward Raman scattering to the $\mathrm{ZnSe}_{0.68} \mathrm{~S}_{0.32}$ zincblende alloy. Due to large optical gaps of $\mathrm{ZnSe}(2.7 \mathrm{eV})$ and $\mathrm{ZnS}(3.6 \mathrm{eV}),{ }^{19} \mathrm{ZnSe}_{0.68} \mathrm{~S}_{0.32}$ is transparent to visible laser lines, thus well-suited in view of a near-forward Raman study. Besides, the frequency gap 


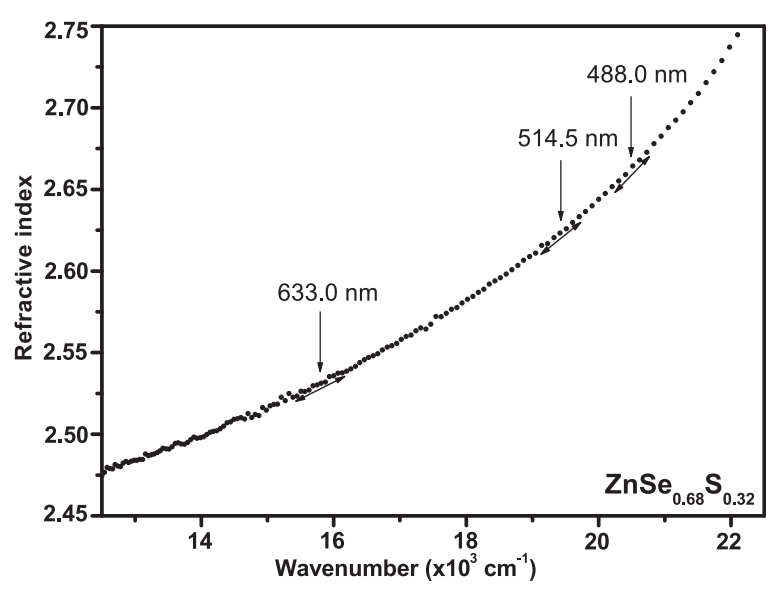

FIG. 2. Ellipsometry measurement of the wavenumber $(\omega)$ dependence of the refractive index $(n)$ of the $\mathrm{ZnSe} 0.68 \mathrm{~S} 0.32$ alloy throughout the visible range. The local dispersion of the refractive index around the laser excitations $488.0,514.5$ and $633.0 \mathrm{~nm}$ are indicated by the corresponding ' $n$ vs. $\omega$ ' slopes, as schematically represented by double arrows.

between the PM-TO's of its $\mathrm{Zn}-\mathrm{Se}$ and $\mathrm{Zn}-\mathrm{S}$ constituting bonds is narrow (235-285 $\mathrm{cm}^{-1}$, see below), with concomitant impact on the magnitude of the $M P^{-}$dispersion (refer to the MP branch in Fig. 1). This offers an opportunity to explore the $M P^{-}$dispersion in a different context than was earlier done with $\mathrm{Zn}_{0.67} \mathrm{Be}_{0.33} \mathrm{Se},{ }^{16}$ the latter alloy being characterized by a comparatively huge $M P^{-}$dispersion $\left(250-450 \mathrm{~cm}^{-1}\right)$. Basically, our aim is to penetrate deep into the $M P^{-}$dispersion of $\mathrm{ZnSe}_{0.68} \mathrm{~S}_{0.32}$ so as to address minimal $q$ values likely to fall into the $L O$-regime of $M P^{-}$, near $\Gamma$.

\section{EXPERIMENT}

Generally, the wavevector $\vec{q}$ of a $T O$ mode (a $P P$ one near $\Gamma$ or the corresponding $P M-T O$ one in the $q_{\infty}$-regime)

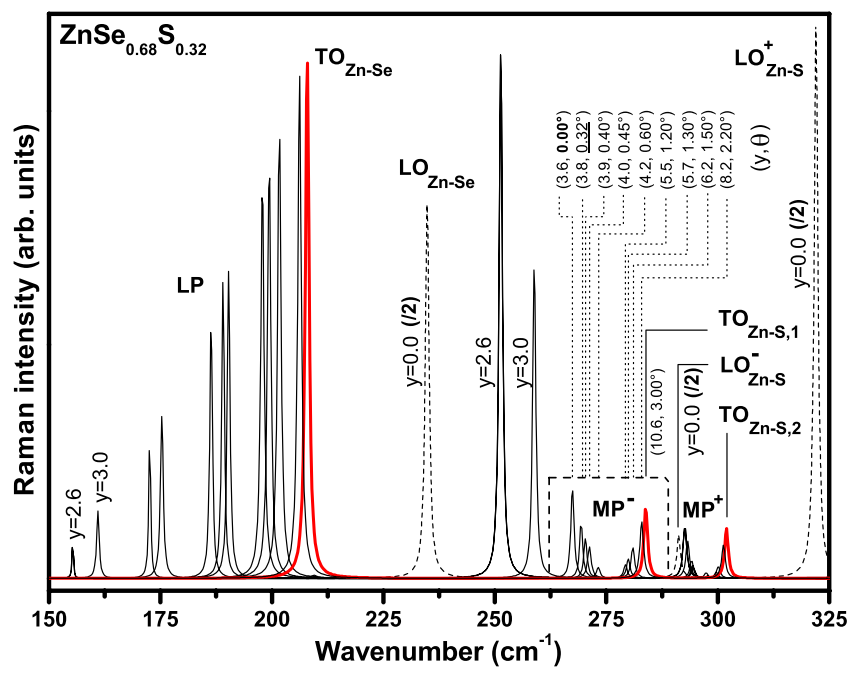

FIG. 3. Theoretical $R C S(y)$ multi- $P P$ near-forward Raman lineshapes of the three-oscillator $[1 \times(\mathrm{Zn}-\mathrm{Se}), 2 \times(\mathrm{Zn}-\mathrm{S})] \mathrm{ZnSe}_{0.68} \mathrm{~S}_{0.32}$ alloy $(p=1-3)$. A uniform phonon damping $\gamma_{p}(x)$ of $1 \mathrm{~cm}^{-1}$ is taken, for a clear resolution of individual features. The $y$ value and corresponding $\theta$ angle for the relevant $M P^{-}$ peaks addressed in the near-forward Raman spectra reported in Figs. 4 and 5 , are indicated within brackets (refer to the dotted box). The theoretical (in bold) and experimental (underlined) $\theta$-limits are emphasized in the reported $(y, \theta)$ values. A simple $T O$-labeling is used for the $P M$-TO's of the reference backscattering signal (in bold-red). accessible in a Raman experiment is governed by the conservation rule $\vec{q}=\vec{k}_{i}-\vec{q}_{s}$ in which $\vec{k}_{i}$ and $\vec{k}_{s}$ are the wavevectors of the incident laser beam and of the scattered light, respectively, both taken inside the crystal, forming an angle $\theta$ therein. In a standard backscattering geometry $\vec{k}_{i}$ and $\vec{k}_{s}$ are (nearly) antiparallel $\left(\theta \sim 180^{\circ}\right)$, so that $q$ is maximum, falling deep into the $q_{\infty}$-regime. Minimum $q$ values, i.e., those likely to address the $P P$-regime, are achieved by taking $\vec{k}_{i}$ and $\vec{k}_{s}$ (nearly) parallel $\left(\theta \sim 0^{\circ}\right)$, using a non-standard nearforward Raman setup. From the above conservation rule,

$$
\begin{aligned}
q= & c^{-1} \times\left\{n^{2}\left(\omega_{i}, x\right) \times \omega_{i}^{2}+n^{2}\left(\omega_{s}, x\right) \times \omega_{s}^{2}-2 \times n\left(\omega_{i}, x\right)\right. \\
& \left.\times n\left(\omega_{s}, x\right) \times \omega_{i} \times \omega_{s} \times \cos \theta\right\}^{0.5}
\end{aligned}
$$

where $\omega_{i}$ and $\omega_{s}$ are the frequencies of the incident and scattered lights, respectively, and $n\left(\omega_{i}, x\right)$ and $n\left(\omega_{s}, x\right)$ the corresponding refractive indexes of the considered $\mathrm{AB}_{1-\mathrm{x}} \mathrm{C}_{\mathrm{x}}$ alloy. If we refer to pure $\mathrm{ZnSe}$ and pure $\mathrm{ZnS}$, the refractive index of $\mathrm{ZnSe}_{1-\mathrm{x}} \mathrm{S}_{\mathrm{x}}$ is expected to decrease with the frequency in the visible range. Accordingly one cannot achieve $q=0(\Gamma)$ in practice. Indeed the minimum $q$ value, accessed in a perfect forward scattering experiment $\left(\theta \sim 0^{\circ}\right)$, i.e., $q_{\min }=\mid n\left(\omega_{i}, x\right) \times \omega_{i}$ $-n\left(\omega_{s}, x\right) \times \omega_{s} \mid$, remains finite because the difference in frequencies is augmented by the difference in refractive indexes. Optimum conditions are thus reached by minimizing the dispersion of the refractive index around the used laser excitation. The alloy composition $(x=0.32)$ as well as the laser excitation $\left(\omega_{i}\right)$ used, were selected in this spirit, as discussed below.

The $\mathrm{ZnSe}_{0.68} \mathrm{~S}_{0.32}$ sample considered in this work was grown from the melt as a single crystal (cylinder, $\sim 3 \mathrm{~mm}$ high and $\sim 8 \mathrm{~mm}$ in diameter) by using the high-pressure Bridgman method (see detail, e.g., in Ref. 20). The selected alloy corresponds to the highest achievable $\mathrm{S}$ incorporation by the Bridgman method. This provides optimal conditions with respect to the dispersion of the refractive index, since the latter is larger for $\mathrm{ZnSe}$ than for $\mathrm{ZnS}$ in the visible range. $^{21}$ In fact, the wavelength $(\lambda)$ dependence of the $\mathrm{ZnSe}_{0.68} \mathrm{~S}_{0.32}$ refractive index $(n)$ measured by ellipsometry throughout the visible range, transposed into a wavenumber $(\omega)$ dependence in Fig. 2, can be accurately fitted to the Cauchy dispersion formula,

$$
n(\lambda)=X+Y \times \lambda^{2} \times 10^{4}+Z \times \lambda^{-4} \times 10^{9}
$$

using $(X, Y, Z)$ values of $(3.1638 \mp 0.0007,1.2380 \mp 0.0536$, $14.0485 \mp 0.1056)$, where $X$ is dimensionless, $Y$ and $Z$ are constants with units of $\mathrm{nm}^{2}$ and $\mathrm{nm}^{4}$, respectively, and $\lambda$ is in $n m$. The minimum dispersion of the $\mathrm{ZnSe}_{0.68} \mathrm{~S}_{0.32}$ refractive index is further achieved by shifting the Raman analysis to the less energetic end of the visible spectral range. We accordingly use the $633.0 \mathrm{~nm}$ line from a He-Ne laser to record the $\mathrm{ZnSe}_{0.68} \mathrm{~S}_{0.32}$ near-forward Raman spectra, instead of the $514.5 \mathrm{~nm}$ and $488.0 \mathrm{~nm} \mathrm{Ar}+$ ones earlier used with $\mathrm{Zn}_{0.67} \mathrm{Be}_{0.33} \mathrm{Se},{ }^{16}$ notwithstanding its inferior Raman efficiency. Along the same line, the Stokes experiment $\left(\omega_{i}>\omega_{s}\right)$ was preferred against the anti-Stokes one $\left(\omega_{i}<\omega_{s}\right)$, not to mention that the Stokes process is, further, more efficient. The near-forward and backward Raman spectra are taken 
along the [110]-growth axis of the sample, corresponding to a nominal ( $T O$-allowed, $L O$-forbidden) geometry, after optical polishing of the opposite (110) faces to optical quality until quasi parallelism was achieved.

\section{RESULTS AND DISCUSSION}

Preliminary insight as to whether there is any chance to access experimentally the presumed $L O$-like $M P^{-}$reinforcement with $\mathrm{ZnSe}_{0.68} \mathrm{~S}_{0.32}$ in a near forward Raman scattering experiment using the $633.0 \mathrm{~nm}$ laser excitation is achieved by calculating the related multi- $P P$ near-forward Raman cross section $(R C S)$ in its $(q, \theta)$-dependence. For doing so we use the generic $R S C$ expression established in Ref. 16 which we reproduce hereafter in its specific form applying to multi$P P^{\prime}$ 's propagating in the bulk of an alloy containing $p$ oscillators (in reference to the multi $P M-T O^{\prime} \mathrm{s}$ of the $q_{\infty}$-regime behind the $P P^{\prime}$ 's),

$$
\begin{aligned}
R C S(\omega, q, x) \sim & \operatorname{Im}\left\{-\left[\varepsilon_{r}(\omega, x)-q^{2} \times c^{2} \times \omega^{-2}\right]^{-1}\right. \\
& \times\left[1+\sum_{p} C_{p}(x) \times L_{p}(\omega, x)\right]^{2} \\
& +\sum_{p} C_{p}^{2}(x) \times \omega_{p}^{2}(x) \times L_{p}(\omega, x) \\
& \left.\times S_{p}^{-1}(x) \times \varepsilon_{\infty, p}^{-1} \times \omega_{p}^{-2}\right\} .
\end{aligned}
$$

$\varepsilon_{r}(\omega, x)$ is the relative dielectric function of the $\mathrm{AB}_{1-\mathrm{x}} \mathrm{C}_{\mathrm{x}}$ alloy taken in its classical form, including, on the one hand, the electronic contribution, $\varepsilon_{\infty}(x)$, varying linearly with $x$ between the parent values, and, on the other hand, a summation over the $p$ ionic oscillators present in the crystal, each represented by a damped Lorentzian resonance $L_{p}(\omega, x)=$ $\omega_{p}^{2}(x) \times\left[\omega_{p}^{2}(x)-\omega^{2}-j \times \gamma_{p}(x) \times \omega\right]^{-1}$ at the relevant $P M$ $T O$ frequency $\omega_{p}(x)$ in the $q_{\infty}$-regime. The damping parameter $\gamma_{p}(x)$, which determines the Raman linewidth, is sample-dependent. $C_{p}(x)$ and $S_{p}(x)$ are the $p$-related FaustHenry coefficient and oscillator strength, monitoring the Raman intensity and the (PM-TO)-LO band of oscillator $p$, respectively. Both parameters scale linearly with the fraction of oscillator $p$ in the crystal. The $\left(C_{p}, S_{p}, \omega_{p}, \varepsilon_{\infty, p}\right)$ values of the pure $\mathrm{ZnSe}$ or $\mathrm{ZnS}$ compound related to oscillator $p$ are $\left(-0.7,2.92,254.5 \mathrm{~cm}^{-1}, 5.75\right)^{16}$ and $\left(-0.45,{ }^{22} 2.57,{ }^{23}\right.$ $277.0 \mathrm{~cm}^{-1},{ }^{15} 5.20$ - see Ref. 15), respectively. Remaining alloy-related input parameters are the number $p$ of oscillators per alloy, the corresponding $\omega_{p}(x)$ frequencies and the fractions $f_{p}(x)$ of such oscillators at a given composition $x$. Such $\left[p, \omega_{p}(x), f_{p}(x)\right]$ parameters refer to the $q_{\infty}$-regime and are thus directly accessible from a pure-TO backscattering Raman insight, or by infrared absorption.

Vinogradov et al. have revealed a well-resolved threemode $[1 \times(Z n-S e), 2 \times(Z n-S)] P M-T O$ pattern $(p=3)$ for $\mathrm{ZnSe}_{1-\mathrm{x}} \mathrm{S}_{\mathrm{x}}$ in their recent exhaustive infrared study. ${ }^{15}$ The question as to whether the $\mathrm{Zn}-\mathrm{S}$ doublet falls into the scope of the 1 -bond $\rightarrow 2$-mode percolation scheme, or not, will be debated elsewhere. For our present use, we may only retain that the two $\mathrm{Zn}-\mathrm{S} P M-T O$ 's, presently labelled as $T O_{Z n-S, 1}$ and $T O_{Z n-S, 2}$ show up distinctly in the backscattering Raman spectrum of $\mathrm{ZnSe}_{0.68} \mathrm{~S}_{0.32}$, being characterized by comparable Raman intensities (a direct insight is given below). This means that the available $\mathrm{Zn}-\mathrm{S}$ oscillator strength, which scales as the $\mathrm{Zn}-\mathrm{S}$ bond fraction, equally shares between the two Zn-S sub-modes, leading to $f_{Z n-S, 1}=f_{Z n-S, 2}=0.16$. As for the unique $\mathrm{Zn}$-Se mode, its oscillator strength directly scales as the $\mathrm{Zn}-\mathrm{Se}$ bond fraction, so that $f_{\mathrm{Zn}-S e}=0.68$. Besides, the three $\left[T O_{Z n-S e}, T O_{Z n-S, 1}, T O_{Z n-S, 2}\right] P M-T O$ 's emerge at the $\omega_{p}(x)$ Raman frequencies of $(\sim 210, \sim 285$, $\sim 303) \mathrm{cm}^{-1}$, correspondingly.

The theoretical $q$-dependent near-forward multi- $P P$ Raman lineshapes derived for $\mathrm{ZnSe}_{0.68} \mathrm{~S}_{0.32}$ via Eq. (3) using the above $\left[p, \omega_{p}(x), f_{p}(x)\right]$ input parameters are shown in Fig. 3, taking a uniform phonon damping of $1 \mathrm{~cm}^{-1}$, for optimum resolution of the individual features. In fact, instead of $q$ we conveniently use the dimensionless parameter $y=q \times c \times \omega_{1}^{-1}$, arbitrarily taking $\omega_{1}$, the $P M-T O$ frequency of the pure $\mathrm{ZnSe}$ crystal in the $q_{\infty}$-regime, for the change of variable. As anticipated, in the theoretical $\operatorname{RCS}(y)$ curves the initial $M P^{-}$collapse at large $y$ values is relayed by a $L O$-like $M P^{-}$reinforcement at moderate-to-small $y$ values (refer to the dotted box). Interestingly, a moderate penetration into the $M P^{-}$dispersion curve, not exceeding one third of the total dispersion (covering the $L O_{Z n-S e}-T O_{Z n-S, 1}$ band, see Fig. 3), seems sufficient to access the $L O$-like reinforcement regime. The crucial question is whether the latter regime can be accessed experimentally, or not. Basically this depends on the dispersion of the refractive index around the used laser excitation, as already discussed (see Sec. II). For a direct insight we indicate in front of a given theoretical $M P^{-}$ peak in Fig. 3 (in reference to the dotted box therein), corresponding to a certain $\omega=\left|\omega_{i}-\omega_{s}\right|$ frequency at a certain $y$ value, the relevant $\theta$ angle for the used $633.0 \mathrm{~nm}$ laser excitation, as directly inferred from Eq. (1) via Eq. (2). As apparent in Fig. 3 the minimum accessible $y$ value with the latter laser line, corresponding to $\theta=0^{\circ}$, hopefully falls into the $M P^{-}$reinforcement regime. This is encouraging in view of a possible detection by near-forward Raman scattering using the $633.0 \mathrm{~nm}$ excitation.

We turn to experiment. For reference purpose, we show at the top of Fig. 4 (thick spectrum) the nominally pure-TO Raman spectrum taken in a backscattering geometry along the [110]-growth crystal axis using the most energetic laser line at hand, namely the $488.0 \mathrm{~nm}$ one. This line is more absorbed than the $633.0 \mathrm{~nm}$ one in our sample. The spurious near-forward Raman signal due to reflection of the incident laser at the back surface of the (quasi) transparent sample is thus minimized. Along with the nominal triplet $\left(\mathrm{TO}_{\mathrm{Zn} \text {-Se }}\right.$, $\left.T O_{Z n-S, 1}, T O_{Z n-S, 2}\right)$ of $P M-T O$ 's described above, several theoretically forbidden features are observed, such as the $L O_{Z n \text { - }}$ Se and $L O_{Z n-S}^{+}$modes at $\sim 245$ and $\sim 322 \mathrm{~cm}^{-1}$, respectively, and also the so-called $A$-band around $165 \mathrm{~cm}^{-1}$, reflecting the acoustical two-phonon density of states. We mention that the emergence of theoretically forbidden optic modes or acoustic bands is a common feature of alloys, due to a partial breaking of the wavector conservation rule [i.e., Eq. (1)] by the alloy disorder. As for the minor $L O_{Z n-S}^{-}$mode of the $\mathrm{Zn}$ $S$ doublet, this remains screened by the nearby $T O_{Z n-S, 1}$ and $T O_{Z n-S, 2}$ PM-TO's (see Fig. 3). Satisfactory contour modeling of the nominal three-mode $P M-T O$ pattern (top thin curve of Fig. 4) is achieved both in the $\mathrm{Zn}-\mathrm{Se}$ (not shown) 


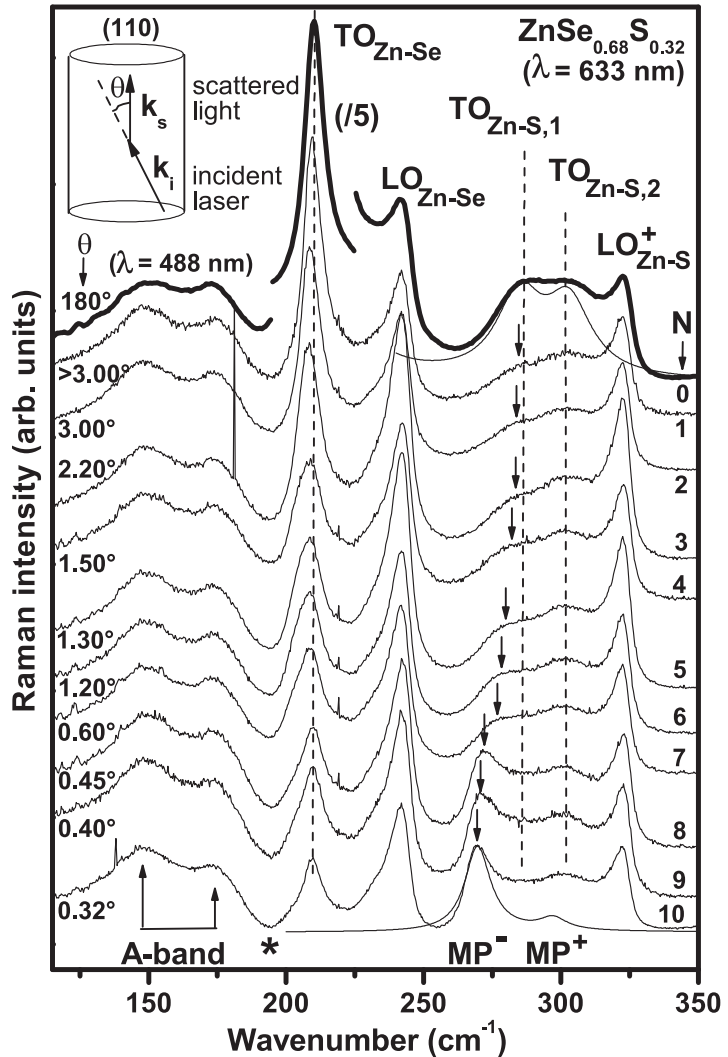

FIG. 4. Intensity-normalized $\mathrm{ZnSe}_{0.68} \mathrm{~S}_{0.32}$ Raman spectra (numbered ' $N$ ' $=1-10)$ taken with the $633.0 \mathrm{~nm}$ laser excitation by using a nearforward scattering geometry, as schematically represented. The nominal/ near-forward (MP labeling) and parasitical/backward (a simple TO labeling is used for the reference $P M-T O$ 's - marked by dashed lines) Raman signals are distinguished. The $\mathrm{ZnSe}_{0.68} \mathrm{~S}_{0.32}$ backward Raman spectrum taken with the $488.0 \mathrm{~nm}$ laser excitation is added (top-thick curve ' $\mathrm{N}$ ' $=0$ ), for reference purpose. The theoretical (thin lines) backward $\left(\theta \sim 180^{\circ}\right)$ and near-forward $\left(\theta \sim 0.32^{\circ}\right) \mathrm{Zn}-\mathrm{S}$ Raman signals are superimposed onto the corresponding experimental ones, for a comparison at extreme $\theta$ angles.

and $\mathrm{Zn}-\mathrm{S}$ (thin line) spectral ranges by injecting the selection of input parameters specified above into the asymptotic form of Eq. (3) valid in the $q_{\infty}$-regime (backscattering-like), in fact reduced to its second term, taking the same $\gamma_{p}(x)$ value of $24 \mathrm{~cm}^{-1}$ for the two $\mathrm{Zn}-\mathrm{S}$ sub-modes.

Now, we change the Raman setup from backscattering to near-forward scattering. A representative series of nearforward Raman spectra taken at different $\theta$ angles with respect to the [110]-growth axis of the $\mathrm{ZnSe}_{0.68} \mathrm{~S}_{0.32}$ ingot using the $633.0 \mathrm{~nm}$ laser line are shown in Fig. 4 (thin spectra). The intensities, normalized to the $\theta$-insensitive A-band, are directly comparable. In each spectrum the near-forward Raman signal ( $M P$-labeling in Figs. 3 and 4$)$ is partially obscured by the $\theta$-insensitive backscattering one (TO-labeling in abbreviation of $P M-T O$ ) generated after reflection of the laser beam at the top surface (detector side) of the sample. As $M P^{+}$is located within the $\mathrm{Zn}-\mathrm{S}$ doublet of parasitical $P M-T O$ 's (see Fig. 3), it remains partially obscured at any angle. The best resolved $P P$ feature is $M P^{-}$, because it falls outside the doublet (refer to the dotted box in Fig. 3). Its $(q, \theta)$-dependence is discussed in detail hereafter.

The relevant $\theta$ angle for each near-forward Raman spectrum of Fig. 4 is determined via a combined adjustment, by

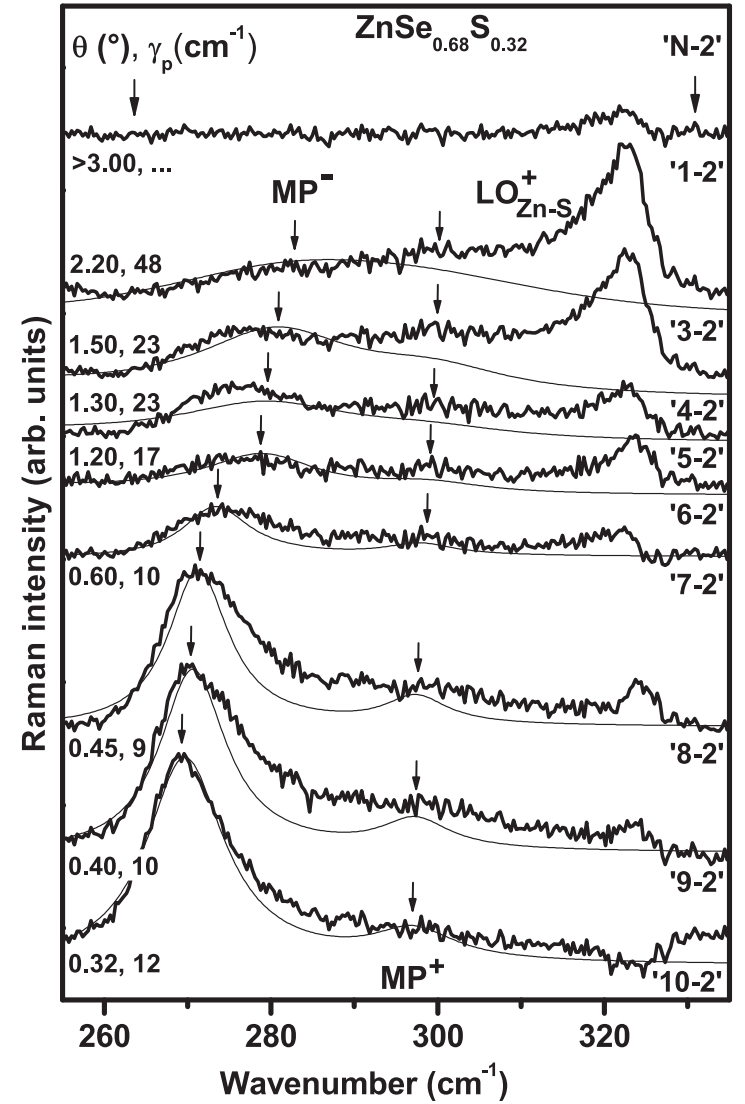

FIG. 5. Representative $P P$-like difference Raman spectra (noted "N-2") obtained by subtracting the parasitical backscattering-like Raman signal (in reference to spectrum "2" of Fig. 4) from the alternative near-forward Raman spectra of the series (with number "N" in Fig. 4). The best-adjusted theoretical PP Raman lineshapes (thin lines) obtained via Eq. (3) by using the indicated $\left[\theta, \gamma_{p}(x)\right]$ values are superimposed, for comparison.

using Eq. (3), of both the frequency and the total area (Raman intensity) of the experimental $M P^{-}$Raman peak (schematically indicated by arrows). A reliable insight into the latter peak, and more generally into the whole $P P$ signal, i.e., including both $M P^{-}$and $M P^{+}$, requires to remove the parasitical contribution from the backscattering contribution in the spectral range of interest. This is achieved by subtracting to each raw near-forward Raman spectrum (numbered "N" in Fig. 4), the upper spectrum of the series (numbered "2"), corresponding to the largest reported $\theta$ angle. The resulting series of ("N-2") $P P$-like difference Raman spectra is shown in Fig. 5. Note that the "1-2" difference spectrum (upper curve) is flat. This indicates that the near-forward Raman spectrum numbered "2" assimilates with the nominal backscattering one, in reference to the upper-thick curve of Fig. 4, even though the analogy between the two spectra is not so obvious. This is due to a difference between the $\gamma_{p}(x)$ values in the backward $\left(\sim 24 \mathrm{~cm}^{-1}\right.$, see above) and nearforward (roughly double, see below) scattering geometries.

The best-adjusted $P P$ theoretical Raman lineshapes (thin lines) obtained via Eq. (3) by using the input parameters specified above, are superimposed onto the corresponding $P P$ difference Raman spectra in Fig. 5-and also in Fig. 4 in the particular case of the smallest achievable $\theta$ angle experimentally, for comparison. Globally, the agreement is rather 
fair, especially if we realize that only one adjustable parameter, i.e., the angle $\theta$, governs both the Raman frequencies (indicated by arrows in Fig. 5) and the Raman intensities of both $M P^{-}$and $M P^{+}$. The only remaining adjustable parameter is the phonon damping $\gamma_{p}(x)$, taken identical for both modes. The relevant $\theta$ and $\gamma_{p}(x)$ values per near-forward Raman spectrum are indicated in Fig. 5 (and also in Fig. 4 regarding $\theta$ ).

Basically, as long as $\theta$ remains larger than $\sim 2.20^{\circ}$, the near-forward Raman signal of $\mathrm{ZnSe}_{0.68} \mathrm{~S}_{0.32}$ approximates to the backscattering one $\left(\theta \sim 180^{\circ}\right)$ and remains stable, as already discussed. Below this critical angle, the multi-TO's enter their $P P$-regime, signed by a red-shift of $M P^{-}$(see arrows in Figs. 4 and 5). Note that the minimum achievable $\theta$ angle remains finite $\left(\sim 0.32^{\circ}\right)$, meaning that the nearforward Raman study cannot be fully developed in practice. This, we attribute to slight $\overrightarrow{k_{i}}$-disorientation inside the crystal due to inherent defects in an alloy. Nevertheless, the accessible $\theta$-domain suffices to reach the $L O$-like reinforcement regime $\left(\theta: 0.60^{\circ} \rightarrow 0.32^{\circ}\right)$ beyond the initial collapse regime $\left(\theta: 3.00^{\circ} \rightarrow 1.20^{\circ}\right)$, testified by the development of $M P^{-}$into a sharp and intense feature at near-normal incidences. An interesting trend regarding $\gamma_{p}(x)$, is that it reduces dramatically with $\theta$. A maximum value of $\sim 48 \mathrm{~cm}^{-1}$ is detected just when departing from the backscattering regime $\left(\theta=2.20^{\circ}\right)$, being roughly reduced by half in the collapse regime, and again roughly by half when penetrating the reinforcement

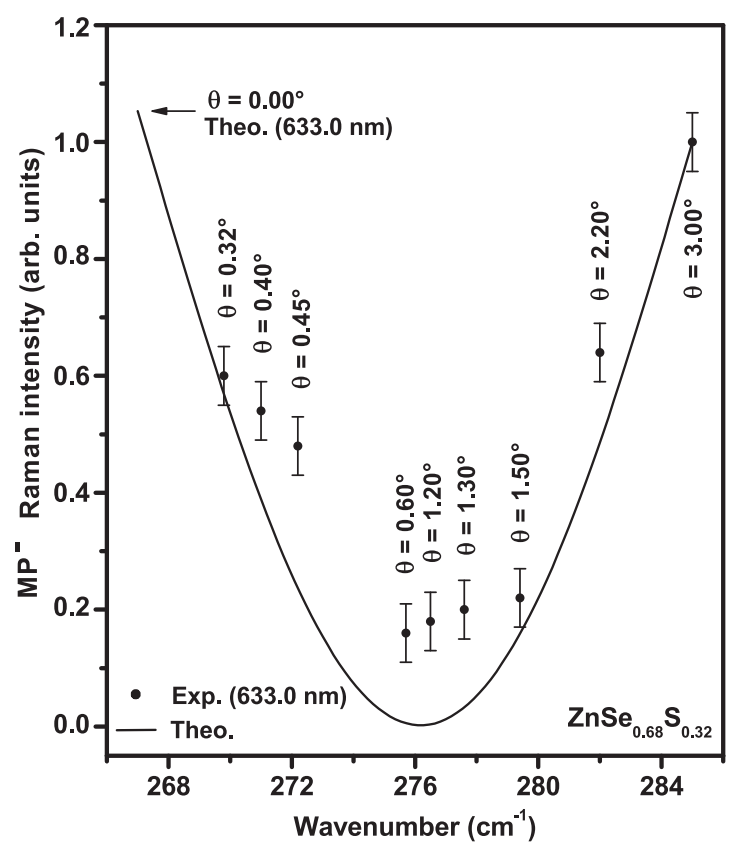

FIG. 6. Comparison between the theoretical [thin line, derived from Eq. (3)] and experimental (symbols) $M P^{-}$Raman intensities depending on $(\omega, \theta)$. The experimental data are spoilt by error bars representing a variation in the estimate of the $M P^{-}$Raman intensity depending on the procedure used to remove the $M P^{+}$and $L O_{Z n-S}^{+}$features (i.e., by using Lorentzian functions, Gaussian functions or a combination of both) from the $P P$-like difference Raman spectra reported in Fig. 5, prior contour modeling of the remaining experimental $M P^{-}$signal. The Raman intensities of the reference experimental and theoretical $T O_{Z n-S, 1}$ signals, from which $M P^{-}$proceeds, are arbitrarily normalized to unity, for a straightforward comparison of the two data sets. regime, with concomitant impact on the $M P^{-}$lifetime (correspondingly increased).

Now, we focus on the $M P^{-}$Raman intensity, of central interest in this work. We display in Fig. 6 the experimental (symbols) and theoretical (thin curve) $(\omega, \theta)$-dependencies of the $M P^{-}$Raman intensities. The reference Raman intensity of the $T O_{Z n-S, 1}$ mode observed in the backscattering geometry (in reference to spectrum " 0 " of Fig. 4), from which the $M P^{-}$proceeds, is arbitrarily normalized to unity, and also the Raman intensity of the corresponding theoretical feature, for a straightforward comparison of the two data sets. Experimentally, the $M P^{-}$Raman intensities are estimated from the ("N - 2") $P P$-like difference Raman spectra reported in Fig. 5, after subtraction of the nearby $M P^{+}$and $L O_{Z n-S}^{+}$modes, and subsequent integration of the remaining $M P^{-}$signal. The experimental data are spoilt by a fixed error bar representing slight differences in the final estimate of the $M P^{-}$Raman intensity depending on the option used to remove the nearby $\mathrm{MP}^{+}$and $L \mathrm{O}_{\mathrm{Zn}-S}^{+}$features (i.e., by using Lorentzian functions, Gaussian functions or a combination of both). As for the theoretical $M P^{-}$Raman intensities, these were obtained by re-calculating the $P P$ Raman lineshapes at the relevant $\theta$ angles by using a phonon damping sufficiently small to generate well-separated $M P^{-}$and $M P^{+}$featuresthe remaining input parameters being otherwise kept constants and by performing a selective curve-fitting of the distinct $M P^{-}$lines (using a Lorentzian function).

When $\theta$ reduces the theoretical trend in Fig. 6 (and also within the hatched box in Fig. 3), corresponding to a dramatic collapse of the $M P^{-}$mode until quasi total extinction, relayed by a strong reinforcement at the approach of the near-normal incidence, is apparent in the experimental data as well, as ideally expected. Only, the latter data generally overestimate the theoretical estimates, i.e., by $10-20 \%$. This might relate to an intrinsic inability in our theoretical approach to account for observed asymmetries of the experimental PP Raman lineshapes (as clearly apparent for most $\theta$ values in Fig. 5).

We have checked that the $L O$-like reinforcement regime of $M P^{-}$is likewise accessible with the alternative $\mathrm{ZnSe}_{0.78} \mathrm{~S}_{0.22}$ alloy (not shown), corresponding to a smaller $\mathrm{S}$ content, meaning that the trend is reproducible. Nevertheless, the emergence of $M P^{-}$at the minimum accessible $\theta$ angle with the latter alloy is less pronounced than with the present $\mathrm{ZnSe}_{0.68} \mathrm{~S}_{0.32}$ alloy, as expected due to the less favorable dispersion of the refractive index (see Sec. II). In contrast, a similar near-forward Raman study using the $633.0 \mathrm{~nm}$ laser excitation performed with the $\mathrm{Zn}_{0.67} \mathrm{Be}_{0.33} \mathrm{Se}$ alloy, earlier studied in Ref. 16, just falls short of engaging the $M P^{-}$reinforcement regime, due to an even less favorable dispersion of the refractive index.

Last, we discuss briefly the $\mathrm{Zn}$-Se signal. At first sight the latter remains $\theta$-insensitive, pinned by a Fano interference with the $A$-band nearby (a characteristic antiresonance is marked by an asterisk in Fig. 4), as earlier discussed for $\mathrm{Zn}_{0.67} \mathrm{Be}_{0.33} \mathrm{Se}{ }^{16}$ The dramatic weakening of the nearforward $\mathrm{Zn}$-Se signal with respect to the backscattering one (divided by $\sim 5$, see Fig. 4) is attributed to a reinforcement of the Fano interference that occurs when the $\mathrm{Zn}$-Se mode 


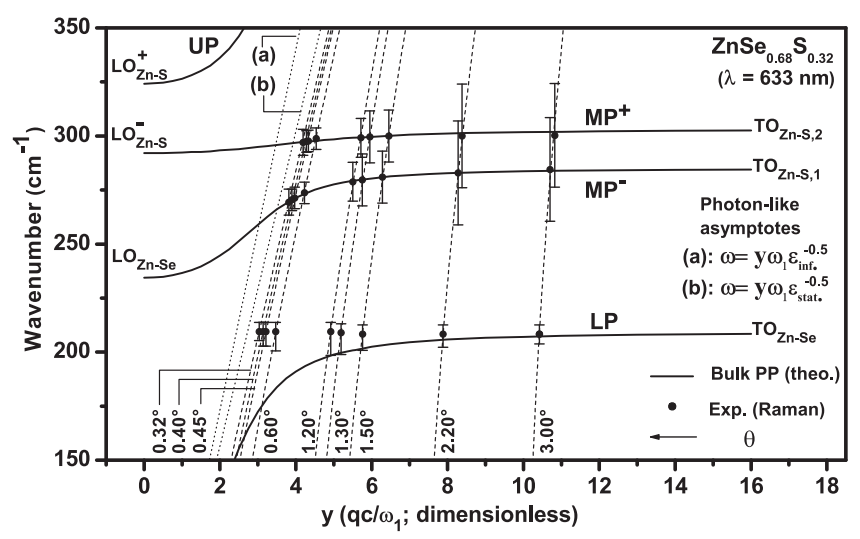

FIG. 7. Comparison between the theoretical and experimental ' $\omega$ vs. $q$ ' multi- $P P$ dispersions of the three-mode $[1 \times(\mathrm{Zn}-\mathrm{Se}), 2 \times(\mathrm{Zn}-\mathrm{S})]$ $\mathrm{ZnSe}_{0.68} \mathrm{~S}_{0.32}$ alloy, as obtained via Eq. (3) (thick lines) and by near-forward Raman scattering (symbols, with error bars representing the Raman linewidths at half height), respectively. The relevant ' $\theta$ vs. $y$ ' correspondences per Raman spectrum, in reference to Eq. (1), are added (refer to the obliquehatched lines), for sake of completeness. The phonon asymptotes in the $q_{\infty}$ regime (using a TO labeling in abbreviation of $P M-T O$ 's, ) and near $\Gamma$ ( $L O$ labeling), together with the photon ones (thin lines) far beyond (a) and far below (b) the $(P M-T O)-L O$ phonon resonances are also specified. The resulting four-mode (lower- $L P$, lower-medium- $M P^{-}$, upper-medium- $M P^{+}$, upper$U P$ ) phonon-polariton pattern can be compared to the more crude threemode MREI-like version displayed in Fig. 1.

engages its $P P$ regime and starts to red-shift towards the $\theta$ insensitive $A$-band. Now, a careful examination reveals that the $\mathrm{Zn}$-Se line develops an increasing asymmetry on its lowfrequency side when $\theta$ reduces, eventually disappearing at near-normal incidences. In the process, the Raman intensity further reduces dramatically. A comparison with the reference $A$-band is explicit with this respect. The discussion of the successive appearance/disappearance of such asymmetrical broadening under $\theta$ reduction, presumably related to some fine structuring of the $\mathrm{ZnSe}$-like $P P$, falls beyond the scope of this work.

For sake of completeness, and for an overview of the obtained results, we provide in Fig. 7 a comparison between the theoretical (thin curves) and experimental (symbols) " $\omega$ vs. $q$ " multi- $P P$ dispersions of $\mathrm{ZnSe}_{0.68} \mathrm{~S}_{0.32}$, as obtained via Eq. (3) - in reference to Fig. 3 and by near-forward Raman scattering - in reference to Figs. 4 and 5 (refer to the arrows), respectively. One may more compare with the more crude MREI dispersion of the same alloy displayed in Fig. 1.

\section{CONCLUSION}

Summarizing, we perform a near-forward Raman study of the three-mode $[1 \times(\mathrm{Zn}-\mathrm{Se}), 2 \times(\mathrm{Zn}-\mathrm{S})] \mathrm{ZnSe}{ }_{0.68} \mathrm{~S}_{0.32}$ alloy in search of the presumed $L O$-like reinforcement of the ( $\mathrm{Zn}$ Se,Zn-S)-mixed $M P^{-}$near $\Gamma$. The laser excitation as well as the alloy compositions were selected so as to minimize the dispersion of the refractive index, a prerequisite to penetrate deep into the $M P^{-}$dispersion in view to address the vicinity of $\Gamma$. The $L O$-regime is successfully accessed, as evidenced by the development of $M P^{-}$into a giant feature at ultimately small scattering angles, solving the raised issue in the positive sense. The discussion is supported by a contour modeling of the $\mathrm{ZnSe}_{0.68} \mathrm{~S}_{0.32}$ multi-PP near-forward Raman lineshapes in their $(q, \theta)$-dependence within the formalism of the linear dielectric response.

\section{ACKNOWLEDGMENTS}

We would like to thank P. Franchetti and J.-P. Decruppe for technical assistance in the Raman measurements, C. Jobart for sample preparation, and A. V. Postnikov for useful discussions and careful reading of the manuscript. This work has been supported by the "Fonds Européens de DEvelopment Régional" of Region Lorraine (FEDER Project No. 34619).

${ }^{1}$ M. Born and K. Huang, in Dynamical Theory of Crystal Lattices (Oxford University Press, Clarendon, 1954).

${ }^{2}$ C. H. Henry and J. J. Hopfield, Phys. Rev. Lett. 15, 964 (1965).

${ }^{3}$ N. S. Stoyanov et al., Nature Mater. 1, 95 (2002).

${ }^{4}$ S. P. S. Porto, B. Tell, and T. C. Damen, Phys. Rev. Lett. 16, 450 (1966).

${ }^{5}$ N. Marschall and B. Fischer, Phys. Rev. Lett. 28, 811 (1972).

${ }^{6}$ D. J. Evans, S. Ushioda, and J. McMullen, Phys. Rev. Lett. 31, 369 (1973).

${ }^{7}$ D. L. Mills and A. A. Maradudin, Phys. Rev. Lett. 31, 372 (1973).

${ }^{8}$ D. L. Mills and E. Burstein, Rep. Prog. Phys. 37, 817 (1974).

${ }^{9}$ J.-I. Watanabe, K. Uchinokura, and T. Sekine, Phys. Rev. B 40, 7860 (1989).

${ }^{10}$ In contrast with the $T O$-like electric field, the $L O$-like electric field suffers no $q$-restriction, and remains invariant (non-dispersive) in between $\Gamma$ and the $q_{\infty}$-regime. Therefore at the latter limit the effective force constant of the $L O$ mode is reinforced by a Colombian contribution with respect to the purely-mechanical force constant of the $P M$-TO mode, meaning that $\omega_{L O}>\omega_{T O}$.

${ }^{11}$ For a given optical mode at $\Gamma$, one may as well consider that the wavevector, nul in fact, is oriented along the atom displacement $(L O)$ or perpendicular to it $(T O)$.

${ }^{12}$ J. Bao and X. X. Liang, J. Phys.: Condens. Matter 18, 8229 (2006).

${ }^{13}$ J. Bao and X. X. Liang, J. Appl. Phys. 104, 033545 (2008).

${ }^{14}$ I. F. Chang and S. S. Mitra, Phys. Rev. 172, 924 (1968).

${ }^{15}$ E. A. Vinogradov, B. N. Mavrin, N. N. Novikova, and V. A. Yakovlev, Phys. Solid State 48, 1940 (2006).

${ }^{16}$ R. Hajj Hussein, O. Pagès, F. Firszt, W. Paszkowicz, and A. Maillard, Appl. Phys. Lett. 103, 071912 (2013).

${ }^{17}$ O. Pagès, J. Souhabi, V. J. B. Torres, A. V. Postnikov, and K. C. Rustagi, Phys. Rev. B 86, 045201 (2012).

${ }^{18}$ A. Bruchhausen, L. M. León Hilario, A. A. Aligia, A. M. Lobos, A. Fainstein, B. Jusserand, and R. André, Phys. Rev. B 78, 125326 (2008).

${ }^{19}$ S. S. Devlin, in Physics and Chemistry of II-VI Compounds, edited by M. Aven and J. S. Prener (North-Holland Publishing Company, Amsterdam, 1967), p. 603.

${ }^{20}$ F. Firszt, A. Wronkowska, A. Wronkowski, S. Łegowski, A. Marasek, H. Meczyńska, M. Pawlak, W. Paszkowicz, J. Zakrzewski, and K. Strzałkowski, Cryst. Res. Technol. 40, 386 (2005).

${ }^{21}$ H. H. Li, J. Phys. Chem. Ref. Data 13, 103 (1984).

${ }^{22}$ This value was adjusted so as to best reproduce the relative intensity ratio between the $\mathrm{Zn}$-Se and $\mathrm{Zn}-\mathrm{S}$ signals in the reference Raman spectrum of $\mathrm{ZnSe}_{0.68} \mathrm{SO}_{.32} 0.32$ should appear as an index taken in the backscattering geometry (see Fig. 4, top thick spectrum).

${ }^{23}$ Value linearly extrapolated for pure $\mathrm{ZnS}$ from best adjustment of the 'Zn-S oscillator strength vs. $x$ ' experimental data reported at moderate $\mathrm{S}$ content for $\mathrm{ZnSe}_{1}-\mathrm{xSx} 1-\mathrm{x}$ and $\mathrm{x}$ should appear as indexes in Ref. 15 (see Fig. 5 therein). 